\title{
Competencias Matemáticas y el éxito de ingreso a la Universidad Nacional de Ingeniería, de los estudiantes del 5 to grado secundaria
}

\section{Matematical abilities and the success of getting a place at Universidad Nacional de Ingeniería, of the secondary 5th grade students}

\author{
Enrique Arturo Delgado Torres ${ }^{1}$
}

\section{RESUMEN}

Objetivo: Identificar la relación de las competencias matemáticas desarrolladas en el éxito de ingreso a la UNI, de los estudiantes de quinto de secundaria. Materiales y Métodos: El estudio es cuantitativo diseño correlacional, se utilizó las notas de ingreso y aplicó un cuestionario a 76 estudiantes, siendo procesados por SPSS, para hallar la correlación se empleó el Rho de Spearman. Resultado: Las variables competencias matemáticas y el éxito de ingreso a la UNI se relacionan $\mathrm{Rho}=, 712$ buena. Asimismo, en cuanto a las hipótesis específicas se pudo comprobar una relación significativa entre la variable competencias matemáticas y las dimensiones autoestima, motivación y conocimiento de las asignaturas, cuya mayoría considera que tienen un nivel adecuado en estas categorías, los cuales son reforzados por las buenas competencias matemáticas de los estudiantes, necesarios para enfrentar un examen admisión. Conclusiones: Las competencias matemáticas se vinculan con el éxito adecuado en el ingreso a la universidad de los estudiantes de nivel secundaria, sin embargo, los puntos de vista de la muestra no representan los máximos niveles.

Palabras clave: Competencias Matemáticas, Éxito, Autoestima, Motivación, Conocimiento.

\begin{abstract}
Objective: To identify the relationship of the mathematical competencies developed in the success of entering the UNI, of the students of the fifth year of secondary school. Materials and Methods: The study is quantitative correlational design, the entrance notes were used and a questionnaire was applied to 76 students, being processed by SPSS, to find the correlation the Spearman Rho was used. Result: The variables mathematical competencies and the success of admission to UNI are related to Rho $=.712$ good. Likewise, regarding the specific hypotheses, a significant relationship could be verified between the variable mathematical competences and the dimensions of self-esteem, motivation and knowledge of the subjects, the majority of which consider that they have an adequate level in these categories, which are reinforced by the good students' mathematical competencies, necessary to face an admission exam. Conclusions: Mathematical competencies are associated with adequate success in college for high school students; however, the points of view of the sample do not represent the highest levels.
\end{abstract}

Keywords: Mathematical Competences, Success, Self-esteem, Motivation, Knowledge

\section{INTRODUCCIÓN}

Según los resultados PISA 2015 el Perú solo alcanzó el nivel 1 en cuanto a las competencias matemáticas que según el MINEDU (2017), menciona. los estudiantes responden preguntas que involucran contextos conocidos, donde encuentran información precisa. Identifican informaciones y utilizan procedimientos rutinarios, siguiendo instrucciones directas. Realizan acciones obvias que se deducen de acuerdo a los estímulos proporcionados. Pero resulta insuficiente, porque las pruebas son diseñadas para lograr nivel 6. Los educandos, adolecen de competencias numéricas para el logro académico superior.

Aunque la Encuesta Nacional de Hogares (ENAHO) realizada en el 2016 y publicado por INEI (2017), señala que solo el 35\% de egresados de secundaria inician una vida académica de nivel superior, es necesario que los colegios cimienten las bases académicas necesarias para que estos puedan enfrentar un examen de admisión, considerando que el acceso, especialmente en las universidades estatales, ya que cuentan con una cantidad de vacantes limitadas. Asimismo, el poder acceder a una vacante también es un reto por la complejidad de los

\section{exámenes.}

La República (2015) realizó una encuesta para conocer las universidades que tienen los exámenes de admisión más difíciles, "los resultados destacan que un 51,3\% piensa que la Universidad Nacional de Ingeniería tiene el examen de admisión más complicado, seguido de un $18,7 \%$ cree que es el de Universidad Nacional Mayor de San Marcos y solo un 3,9 \% cree que la Universidad Católica del Perú tiene el examen más difícil. Con ello, damos cuenta la necesidad de analizar las temáticas que se dictan en clases, para apoyar en su preparación para la universidad".

INEI (2015) destaca que el $43,7 \%$ de los universitarios considera que el prestigio de la universidad es fundamental para seleccionar a una universidad para iniciar una vida académica de nivel superior. En años anteriores, el INEI (2014) incluso identificó las áreas predilectas por jóvenes a partir de 17 años que estudió o estudia educación superior universitaria, entre estas se destaca Educación con un 19,6\%, seguido de Ingeniería con un $18,4 \%$.

De otro lado, también es necesario tomar en cuenta que el éxito de ingreso no solo depende de la preparación académica en general, sino también de la fortaleza emocional y mental de los 
estudiantes, como la autoestima y motivación.

Esta investigación se centra en el desarrollo de las variables Competencias Matemáticas y Éxito de Ingreso, de las cuales ya existían estudios o investigaciones que buscaban explicar el contexto en al que se desarrollan. Como es el caso de Paredes (2012) quien encontró dificultades en los estudiantes de secundaria al momento de resolver ejercicios matemáticos, por ello propuso el método problémico con el cual demostró la posibilidad de fortalecer las competencias en dicho curso, en cuanto a capacidad de razonamiento y demostración, comunicación matemática y resolución de problemas, en otras palabras, la identificación de una metodología facilitará el aprendizaje de la matemática.

Por su parte, Penengo (2015) indica que es muy importante conocer a los estudiantes que ingresan a la universidad, puesto que los problemas se inician cuando los estudiantes no han adquirido los conocimientos suficientes para iniciar cursos de nivel superior. Además, considera que es importante tomar en cuenta las características del momento vital que atraviesa el estudiante y lograr un proceso de adaptación a los cambios y al impacto de las nuevas dinámicas que presenta la institución. En otras palabras, el éxito dependerá de las bases previas que hayan recibido en la escuela para que o tenga inconvenientes con las asignaturas de nivel superior.

El propósito de la investigación es identificar la relación de las competencias matemáticas desarrolladas en el éxito de ingreso a la Universidad Nacional de Ingeniería, de los estudiantes del 5to grado de las Instituciones Educativas Públicas del Nivel Secundario de Lima Metropolitana - 2015, de igual manera se intenta explicar la relación entre sus dimensiones.

\section{MATERIALES Y MÉTODOS}

El estudio es cuantitativo diseño correlacional, se utilizó las notas de ingreso y aplicó un cuestionario a 76 estudiantes. Los resultados fueron procesados con la fórmula de Spearman, para determinar la correlación entre las variables mediante el estadístico SPSS.

\section{RESULTADOS}

Luego del tratamiento estadístico se obtuvieron los siguientes resultados:

\section{Tabla1}

Frecuencias de la variable éxito de ingreso a la universidad

\begin{tabular}{llr}
\hline Exito de ingreso a la universidad & Frecuencia \\
\hline \multirow{2}{*}{ Validos } & Aveces adecuados & 1 \\
& Adecuados & 74 \\
& Muy adecuado & 1 \\
\cline { 2 - 3 } & Toral & 76 \\
\hline
\end{tabular}

El 97,4\% (74 estudiantes) consideraron el éxito para acceder a la universidad como adecuado, el 1,3\% (1 estudiante) refiere como muy adecuado y 1,3\% (1 estudiante) lo califica como a veces adecuado. Esto pone en evidencia el éxito para ingresar a la UNI depende del nivel de preparación sobre todo de las competencias matemáticas desarrolladas en los centros educativos de quinto de secundaria y una preparación preuniversitaria.

\section{Hipótesis General}

HG. Las competencias matemáticas se relacionan con el éxito de ingreso a la UNI, de los estudiantes del 5to grado de secundaria

Tabla 2

Correlación competencias investigativas y éxito de ingreso a la universidad

\begin{tabular}{lllr}
\hline Correlaciones & & Frecuencia \\
\hline $\begin{array}{l}\text { Rho de } \\
\text { spearman }\end{array}$ & $\begin{array}{l}\text { competencia } \\
\text { matematica }\end{array}$ & $\begin{array}{l}\text { Coeficientes de } \\
\text { correlacion }\end{array}$ &, $712 "$ \\
& & Sig. (bilateral) &, 000 \\
\cline { 3 - 3 } & & N &, 76 \\
\hline
\end{tabular}

Se halló una correlación buena de Rho $=, 712$ y el $p=$ 0,000 . Aceptándose la hipótesis alterna, conforme las respuestas de la muestra, quienes en su mayoría consideran las competencias matemáticas se vinculan al éxito de los estudiantes de secundaria que ingresan a la universidad.

\section{Primera Hipótesis Específica}

H1. La autoestima se vincula con el éxito de ingreso a la UNI, de los estudiantes del 5 to grado.

Tabla 3

Correlación competencias investigativas y autoestima

\begin{tabular}{lllr}
\hline Correlaciones & & \multicolumn{2}{c}{ Autestima } \\
\hline $\begin{array}{l}\text { Rho de } \\
\text { spearman }\end{array}$ & $\begin{array}{l}\text { competencia } \\
\text { matematica }\end{array}$ & $\begin{array}{l}\text { Coeficientes de } \\
\text { correlacion }\end{array}$ & \multirow{2}{*}{, 323} \\
\cline { 3 - 3 } & & Sig. (bilateral) &, 004 \\
\cline { 3 - 3 } & $\mathrm{N}$ &, 76 \\
\hline
\end{tabular}

Se encontró una correlación baja de Rho $=, 323$ y el $p=0,000$. Por lo cual se refuta a la hipótesis nula y se acepta la hipótesis alterna, quienes mayoritariamente consideran las competencias matemáticas se vinculan con la autoestima de los estudiantes para ingresar a la universidad.

\section{Segunda Hipótesis Específica}

H2. La motivación docente se vincula con el éxito de ingreso a la UNI, de los estudiantes del 5 to grado de secundaria. 
Tabla 4

Correlación entre competencias Matemáticas y motivación

\begin{tabular}{lllc}
\hline Correlaciones & & Motivación \\
\hline $\begin{array}{l}\text { Rho de } \\
\text { spearman }\end{array}$ & $\begin{array}{l}\text { competencia } \\
\text { matematica }\end{array}$ & $\begin{array}{l}\text { Coeficientes de } \\
\text { correlacion }\end{array}$ &, $712 "$ \\
\hline
\end{tabular}

\begin{tabular}{lr}
\hline Sig. (bilateral) &, 000 \\
\hline $\mathrm{N}$ &, 76 \\
\hline
\end{tabular}

Asimismo, se halló una correlación buena de Rho $=, 712$. Aceptándose la hipótesis alterna, en su mayoría consideran la motivación en los estudiantes para ingresar a la universidad, es importante.

\section{Tercera Hipótesis Específica}

H3. Los conocimientos de las asignaturas se vinculan con el éxito de ingreso a la UNI, de los estudiantes del 5 to grado de secundaria.

\section{Tabla 5}

Correlación entre competencias Matemáticas y conocimiento de las asignaturas

\begin{tabular}{lllr}
\hline \multicolumn{1}{c}{ Correlaciones } & & Motivación \\
\hline $\begin{array}{l}\text { Rho de } \\
\text { spearman }\end{array}$ & $\begin{array}{l}\text { competencia } \\
\text { matematica }\end{array}$ & $\begin{array}{l}\text { Coeficientes de } \\
\text { correlacion }\end{array}$ &, $712 "$ \\
\cline { 3 - 3 } & & Sig. (bilateral) &, 000 \\
\cline { 3 - 3 } & $\mathrm{N}$ &, 76 \\
\hline
\end{tabular}

Se halló Rho $=, 712$. Se acepta la hipótesis alterna, mayoritariamente opinan el dominar las asignaturas juega un papel importante para ingresar a la universidad.

\section{DISCUSIÓN}

Esta investigación permitió identificar la relación de las competencias matemáticas desarrolladas en el éxito de ingreso a la UNI, de los estudiantes del 5to grado de secundaria.

Los resultados pertenecientes a la hipótesis general, representados por las variables competencias matemáticas y el éxito de ingreso a la universidad identificaron una correlación muy buena de $\mathrm{Rho}=, 712$. Esto debido a que la mayoría de la muestra destacó que el $98,7 \%$ de las competencias matemáticas son considerados buenos, lejos de un 1,3\% que lo identifica de muy buena. Por otro lado, un $97,4 \%$ de la muestra califica de adecuado el éxito de ingreso, mientras que 1,3\% lo refiere de muy adecuado.

La primera hipótesis específica encontró una correlación baja de $\mathrm{Rho}=, 323$ representados por la variable competencias matemáticas y la dimensión autoestima. Este resultado estadístico se debe a las opiniones mayoritarias de la muestra con respecto a las competencias matemáticas donde un $98,7 \%$ lo consideran bueno, seguido del $1,3 \%$ referido como muy bueno. Por otro lado, un $86,8 \%$ de la muestra califica de adecuado la autoestima, mientras que el $7,9 \%$ lo refiere de muy adecuado.

En referencia a la segunda hipótesis específica, encontramos una correlación buena de Rho $=, 712$ representados por la variable competencias matemáticas y la dimensión motivación. Este análisis estadístico tomó en cuenta las opiniones mayoritarias de la muestra con respecto a las competencias matemáticas donde un $98,7 \%$ lo considera bueno, seguido del $1,3 \%$ referido como muy bueno. Por otro lado, un $97,4 \%$ de la muestra califica de adecuado la motivación, mientras que 1,3\% lo refiere de muy adecuado.

La tercera hipótesis específica halló una correlación buena de Rho $=, 712$ representados por la variable competencias matemáticas y la dimensión conocimiento de las asignaturas. Esto debido a que la mayoría de la muestra destacó que el $98,7 \%$ de las competencias matemáticas son considerados buenos, lejos de un 1,3\% que lo identifica de muy buena. De otro lado, un $97,4 \%$ de la muestra califica de adecuado el conocimiento de las asignaturas, mientras que el $1,3 \%$ lo refiere de muy adecuado.

Cázares (2015), intentó explicar las competencias matemáticas que el estudiante necesita para afrontar un examen de admisión, en donde destaca la importancia de la programación de los cursos, temáticas y evaluaciones, sin embargo, también destacan que el desempeño de los estudiantes es proporcional al desempeño de los docentes al momento de enseñar, situación que podría suceder pero no forma general, puesto que si el estudiante no tiene el interés de aprender no pondrá atención al docente por más dinámico que sea. Incluso esta investigación identificó que la mayoría de los estudiantes tienen buenas competencias matemáticas, pero aún no alcanzaron sus niveles máximos y eso se debe a que hay aún existen aspectos que mejorar.

Gualtero (2016), analizó las causas por la que los estudiantes de nivel básico no desean continuar estudios superiores, entre otros factores es el temor por afrontar un examen de admisión y la poca motivación para proponerse metas y objetivos, por ello al aplicar un proyecto comprobó la satisfacción de la mayoría de ellos, puesto que pudieron identificar sus habilidades, inteligencias múltiples, destrezas, intereses vocacionales y profesionales, asimismo con el apoyo de talleres de autoestima, esta perspectiva cambió. Esta situación se asemeja a los resultados encontrados en la presente investigación, puesto que nuestra población se encontró consideró que el éxito de ingreso a la universidad es el resultado de una adecuada autoestima, motivación y conocimiento de las asignaturas.

\section{CONCLUSIONES}

A mayor dominio de la competencia matemática, el éxito de ingreso a la universidad de los estudiantes de nivel secundaria, se garantiza.

Un adecuado manejo de la autoestima y motivación en la preparación académica de los estudiantes de quinto de secundaria garantiza el ingreso a la universidad. 
Finalmente, el conocimiento o dominio de las asignaturas ayudan sobre manera alcanzar el éxito de ingreso a la universidad.

\section{REFERENCIAS.}

Cázares, M. (2015). Competencias de Matemáticas de los Estudiantes del Instituto Valladolid Preparatoria de Morelia como aspirantes universitarios (Tesis Posgrado) Universidad de Oviedo, España. $\mathrm{R}$ e c u p e r a d o e n : http://digibuo.uniovi.es/dspace/bitstream/10651/37371/6/T D MarioAlbertoCazarezMeza.pdf

Gualtero, M. (2016). Importancia del proyecto de vida, como eje motivacional para el ingreso a estudios superiores, en los estudiantes de los grados $10^{\circ}$ y $11^{\circ}$, de la Institución Educativa Técnica La Chamba, del GuamoTolima (Tesis Posgrado) Universidad del Tolima, C o l o m bi a. R e c u p e r a d o e n : http://repository.ut.edu.co/bitstream/001/1668/1/IMPORTA NCIA\%20DEL\%20PROYECTO\%20DE\%20VIDA.pdf

La República (7 de diciembre de 2015). Estas son las universidades que tienen los exámenes de admisión más difíciles, según nuestros lectores. Recuperado en: https://larepublica.pe/educacion/724111-estas-son-lasuniversidades-que-tienen-los-examenes-de-admision-masdificiles-segun-nuestros-lectores-fotos
INEI (2017). Perú: Línea de base dé las principales indicaciones disponibles de los objetivos de desarrollo sostenible. Lima: Instituto Nacional de Estadística e I n formát i c a. R e c u p e r a d o e n : https://www.inei.gob.pe/media/MenuRecursivo/publicacion es_digitales/Est/Lib1429/libro.pdf

INEI (2014). Perú: Indicadores de Educación por Departamentos, 2001-2012. Lima: Instituto Nacional de Estadística e Informática.

INEI (2015). Encuesta Nacional a Egresados Universitarios y Universidades, 2014. Principales Resultados. Lima: Instituto Nacional de Estadística e Informática.

MINEDU (2017). El Perú en PISA 2015 Informe nacional de resultados. Lima: Ministerio de Educación

Paredes, A. (2012). Método problémico para desarrollar competencias matemáticas en las alumnas del primero de secundaria de una Institución Educativa del Callao (Tesis Posgrado) USIL, Lima, Perú.

Penengo, V. (2015). El ingreso a la Vida Universitaria. Un recorrido incierto. Montevideo: Universidad de la República de Uruguay. Recuperado en: https://www.colibri.udelar.edu.uy/jspui/bitstream/12345678 9/7842/1/Penengo $\% 2 \mathrm{C} \% 20$ Valentina.pdf 